Revista Ingeniería Biomédica

ISSN 1909-9762 / E-ISSN 1909-9991

Volumen 13 / Número 25 / enero-junio de 2019 / pp. 63-76

Universidad EIA / Envigado, Colombia

\title{
Modelo de gestión para un laboratorio de evaluación de desempeño de tecnología médica
}

\author{
B. E. García ${ }^{1, \psi}$, J. H. García ${ }^{1}$ \\ ${ }^{1}$ Grupo de Investigación en Bioinstrumentación e Ingeniería Clínica - GIBIC, \\ Programa de Bioingeniería, Facultad de Ingeniería, Universidad de Antioquia UdeA
}

Recibido 12 de enero de 2019. Aceptado 18 de mayo de 2019

Resumen-primum non nocere "lo primero es no hacer daño" es uno de los pilares fundamentales de la atención en salud; por lo que disminuir los eventos adversos asociados al uso de equipos y dispositivos médicos es uno de los retos más importantes para las entidades prestadoras de servicios de salud. Lograr esto implica coordinar muchos procesos relacionados entre sí, como son los mantenimientos preventivos, correctivos, control metrológico y validación de tecnología entre otros; esto se dificulta mas cuando el hecho de dar cumplimiento a normas que exigen la realización de pruebas de desempeño se une con la carencia de laboratorios o entidades que realicen este tipo de pruebas a equipos médicos en Colombia. Adicional a esto, en el país se ha incrementado el número de empresas que desarrollan tecnología médica y que para obtener los permisos necesarios para comercialización se encuentran con la falencia de laboratorios y procesos adecuados para certificar dicha tecnología. Para demostrar el funcionamiento adecuado de la tecnología médica se requieren laboratorios que tengan la competencia técnica y capacidad operativa para suplir esta necesidad, y las empresas de desarrollo requieren, además, contar con un laboratorio certificado o acreditado para tal fin.

En Colombia, las universidades cuentan con la infraestructura y dotación para realizar estas evaluaciones, pero no con los métodos o procesos para ser laboratorios de pruebas.

Este trabajo plantea una opción, a través de la creación de un modelo de gestión para el laboratorio del Grupo de Investigación en Bioinstrumentación e Ingeniería Clínica GIBIC del programa de Bioingeniería de la Universidad de Antioquia, en el cual se cuente con los procesos, protocolos, tecnología y personal adecuado para garantizar la realización adecuada de pruebas de desempeño, la metodología incluyó desde la creación del organigrama y la definición de normatividad a cumplir obteniendo finalmente el diseño y validación de protocolos de prueba para diferentes tecnologías.

Palabras clave_Equipo Biomédico, Dispositivos médicos, Ingeniería Clínica, Pruebas de Desempeño, Prueba de Referencia. 


\title{
Management Model For Medical Technology Performance Evaluation LABORATORY
}

\begin{abstract}
Therefore, reducing the adverse events associated with the use of medical equipment and devices is one of the most important challenges for health service providers. Achieving this implies coordinating many related processes, such as preventive, corrective maintenance, metrological control and technology validation among others. This is more difficult when the fact of complying with norms that demand performance tests is combined with the lack of laboratories or entities that perform this type of tests on medical equipments in Colombia. In addition, the number of companies that develop medical technology has increased in the country and, in order to obtain the necessary authorizations for commercialization, there is a lack of laboratories and processes to certify this technology. To demonstrate the proper functioning of medical technology, laboratories that have the technical competence and operational capacity to supply this need are required, and development companies also require a certified or accredited laboratory for this purpose. This work proposes an option, through the creation of a management model for the Bioinstrumentation and Clinical Engineering Research Group GIBIC, in which the processes, protocols, technology and adequate personnel are available to guarantee the adequate performance tests, the methodology included from the creation of the organization chart and the definition of regulations to be met, finally obtaining the design and validation of test protocols for different technologies.
\end{abstract}

Keywords - Biomedical Equipment, Medical Devices, Clinical Engineering, Performance Tests, Benchmark Testing

\section{Modelo DE GERENCIAMENTO PARA UM LABORATÓRIO DE AVALIAÇÃO DE DESEMPENHO DE TECNOLOGIA MÉDICA}

Resumo - primum non nocere "a primeira coisa a não prejudicar" é um dos pilares fundamentais dos cuidados de saúde; Portanto, reduzir os eventos adversos associados ao uso de equipamentos e dispositivos médicos é um dos desafios mais importantes para os prestadores de serviços de saúde. Conseguir isso implica coordenar muitos processos relacionados, como manutenção preventiva, corretiva, controle metrológico e validação de tecnologia, entre outros; Isso fica mais difícil quando o fato de cumprir as normas que exigem testes de desempenho está relacionado à falta de laboratórios ou entidades que realizam esse tipo de teste em equipamentos médicos na Colômbia. Além disso, no país aumentou o número de empresas que desenvolvem tecnologia médica e, para obter as autorizações necessárias para a comercialização, constatam a falta de laboratórios e processos adequados para certificar a referida tecnologia. Para demonstrar o funcionamento adequado da tecnologia médica, são necessários laboratórios com competência técnica e capacidade operacional para atender a essa necessidade, e as empresas de desenvolvimento também exigem um laboratório certificado ou credenciado para esse fim.

$\mathrm{Na}$ Colômbia, as universidades possuem infraestrutura e equipamentos para realizar essas avaliações, mas não os métodos ou processos para testar laboratórios.

Este trabalho levanta uma opção, através da criação de um modelo de gestão para o Grupo GIBIC de Laboratório de Bioinstrumentação e Engenharia Clínica do programa de Bioengenharia da Universidade de Antioquia, no qual os processos, protocolos, tecnologia e pessoal adequado para garantir o desempenho adequado dos testes de desempenho, a metodologia incluída desde a criação do organograma e a definição dos regulamentos a serem cumpridos, obtendo finalmente o design e a validação dos protocolos de teste para diferentes tecnologias.

Palavras-chave — Equipamento Biomédico, Dispositivos Médicos, Engenharia Clínica, Testes de Desempenho, Teste de Referência.

\section{INTRODUCCIÓN}

$\mathrm{D}$ esde los orígenes del ser humano se ha establecido que hacer las cosas bien y de la mejor manera posible proporciona un alto grado de competitividad en el entorno en que se interactúa. Actualmente esto es evidenciado en la implementación de modelos de gestión de calidad basados en procesos donde se establecen y gestionan actividades relacionadas entre sí, identificando la interacción de estas y la gestión realizada para obtener los resultados esperados. La principal característica de los modelos de gestión de calidad incluye la organización completa de personal, departamentos, facultades, activi- dades y procesos. Donde identifican, controlan los grados de calidad que están a cargo de su responsabilidad [1][2]. La organización mundial de la salud establece que: "ES sumamente importante que la seguridad y el desempeño de los dispositivos médicos sean evaluados continuamente cuando estos están en uso, ya que sus características solo pueden ser probadas si se miden cuando un dispositivo funciona bajo ciertas condiciones". Y se establece, además, la importancia de las evaluaciones de desempeño en etapas de pre y posmercado como una responsabilidad compartida entre el fabricante y el tenedor del equipo, y que deberá ser expedida por un laboratorio autorizado para tal fin mediante certificado de acreditación ante la ONAC 
[3]. Como puede comprobarse en el documento emitido por el Ministerio de Salud, el Ministerio de industria y comercio y la Super Intendencia de Industria y Comercio no existen en Colombia laboratorios acreditados para realizar calibración o evaluaciones de desempeño a los equipos objeto de estudio en este trabajo. Al no contar con estos laboratorios en el país, los fabricantes de equipos y dispositivos médicos tienen un obstáculo adicional al proceso de mercadeo, ya que estas pruebas deben ser realizadas por distintos laboratorios acreditados en diferentes magnitudes, con el inconveniente adicional que para la etapa de posmercado, no se cuenta con protocolos de pruebas de desempeño para este tipo de equipos, pero la normatividad exige el mantenimiento, control y seguimiento de los mismos bajo protocolos establecidos y ejecutados bajo altos estándares de competencia técnica [4]. En Colombia, las universidades cuentan con la infraestructura y dotación para realizar estas evaluaciones, pero no con los métodos o procesos para ser laboratorios de pruebas.

En este trabajo se presenta la estructura y el proceso por medio de un modelo de gestión de calidad, para el laboratorio del grupo GIBIC, integrando diversas actividades para llevar a cabo las pruebas de desempeño de la tecnología médica, el cual cuenta con los equipos básicos de evaluación y una infraestructura apta para definir las diferentes zonas que dan cumplimiento a los estándares de calidad y a los requisitos mínimos para alcanzar una certificación, donde se establecen los procesos y sistemas de gestión administrativa para prestar los servicios de pruebas de desempeño para cada una de las tecnologías propias del grupo y las seleccionadas para este proyecto además de un protocolo general mediante el cual se pueden definir los protocolos para una gran variedad de equipos.

\section{Materiales y Métodos}

La revisión bibliográfica se llevó a cabo realizando búsquedas en artículos, libros, tesis, bases de datos, sobre los diferentes protocolos, modelos de gestión de evaluación de desempeño de tecnología médica. Se realizó un recorrido por la literatura definida por los entes reguladores a nivel mundial de tecnología médica y se estudiaron las diferentes metodologías que aplican para que una tecnología pueda entrar al mercado y ser comercializada mediante requisitos técnicos, documentación y diferentes pruebas a las que debe ser sometida antes de que el fabricante pueda comercializarla en una región especifica; para esto se utilizaron bases de datos de entes reguladores internacionales como la FDA y la Unión Europea. Finalmente, como requisito fundamental, se establecieron los estándares principales que determinan los límites operacionales de este tipo de tecnología. Se realizó un estudio del Decreto 4725 de 2005 del INVIMA, para conocer los lineamentos exigidos y establecer el contenido del informe que avale el buen funcionamiento de tecnología médica, para obtener el registro otorgado por ellos para comercializar la tecnología. Se realizó un estudio sobre modelos de gestión, nivel organizacional de personal, cargos, capacidad y estructura física, esto para entender el concepto y poder ser aplicado al laboratorio del grupo GIBIC; también se realizó una búsqueda bibliográfica de las normatividades colombianas existentes que deben ser aplicadas a laboratorios de acuerdo con los procesos que se pretenden realizar en él, es decir, para este caso, pruebas de desempeño, gestión de calidad y almacenamiento de tecnología médica, de esta búsqueda se obtuvo la NTC/ISO 17025:2017 que da los lineamientos para laboratorios de ensayo y calibración.

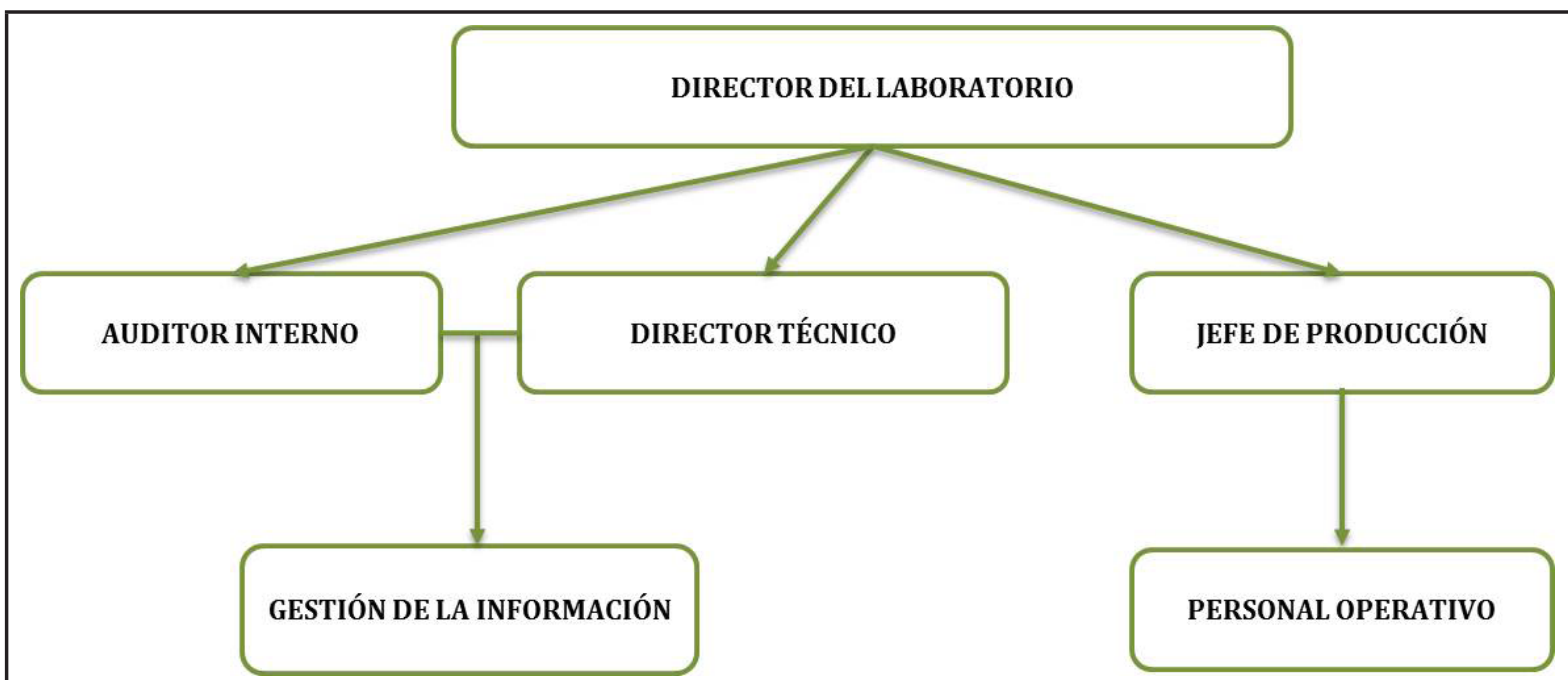

Fig. 1. Organigrama del laboratorio del grupo GIBIC, fuente propia 


\section{Diseño de estructura organizativa y estructura do- cumental del laboratorio del grupo GIBIC}

Una vez obtenida la normatividad técnica que debe ser aplicada al laboratorio del grupo GIBIC para los procesos, se procedió a diseñar la estructura organizativa del mismo, donde se aplicaron los lineamientos de la NTC/ ISO 17025:2017 y se definió la estructura organizativa y documental del laboratorio.

Para el diseño de la estructura organizativa se tuvo presente que el laboratorio pertenece a la Universidad de Antioquia y este diseño se realizó dependiendo de la capacidad de personal del grupo GIBIC de la Universidad, analizando el perfil y respetando los requerimientos de las normas aplicables en este diseño.

Laboratorio de ingeniería clínica del grupo GIBIC: estructura organizativa

Para definir la estructura organizativa del laboratorio del grupo GIBIC se tuvo en cuenta la capacidad de personal con el que esta cuenta para trabajar. En la Fig. 1 se presenta el organigrama del personal.

Para el diseño documental del laboratorio se aplicaron los lineamientos donde se establece que se debe dar cumplimiento a requisitos de gestión y técnicos aplicados a los procesos que se realizan en el laboratorio. Debido a que en el laboratorio del GIBIC, no solo se realizarán pruebas de desempeño, sino que también se pretende realizar toda la gestión de calidad, almacenamiento y etiquetado de las innovaciones del GIBIC, se tuvieron en consideración los requisitos de gestión y técnicos de las normatividades aplicables, es decir NTC/ISO 17025:2017 que da los lineamientos para laboratorios de ensayo y calibración, ISO 13485, gestión de calidad de tecnología médica y la resolución 4002, almacenamiento de tecnología médica [5]-[7].
Diseño y validación de la capacidad del laboratorio del grupo GIBIC encaminando a la certificación o acreditación

Se realizó un estudio de la capacidad física y de las instalaciones del laboratorio del GIBIC, en el que se analizó la ubicación y diseño, de tal forma que fueran apropiadas para los procesos a realizar; además, de que se garantizara la minimización de los riesgos, la adecuada limpieza, el mantenimiento, el orden, evitar la acumulación de agentes contaminantes y toda condición que pueda influir negativamente en la calidad de la tecnología médica.

Se realizó un inventario de los equipos del grupo GIBIC, destinados a realizar las pruebas de desempeño y se definió cuáles iban a ser utilizados en el laboratorio especificando capacidad y magnitud de medida.

Diseño del modelo de gestión para el laboratorio del grupo GIBIC

Posteriormente al diseño de la capacidad organizativa, de personal y documental del laboratorio, se diseñó el modelo de gestión del laboratorio. Para el diseño del modelo se definieron variables como: normas aplicables, personal y pruebas de desempeño de tecnología médica.

Según los procesos que se realizaran en el laboratorio, las normas aplicables son la NTC 17025:2017, la ISO 13485 y el decreto 4002. El personal está compuesto por las directivas de la Universidad de Antioquia y del personal encargado del laboratorio para definir la infraestructura adecuada, donde sus funciones son vigilar que el personal tenga una constante capacitación en cada uno de los procesos que se realizarán e implementar los protocolos de pruebas de desempeño de tecnología médica. Todo esto con el fin de alcanzar la certificación y/o acreditación de laboratorio.

A continuación, en la Fig. 2 se muestra el diseño del modelo de gestión del laboratorio con las normativas que se tuvieron en cuenta [5][6][7].

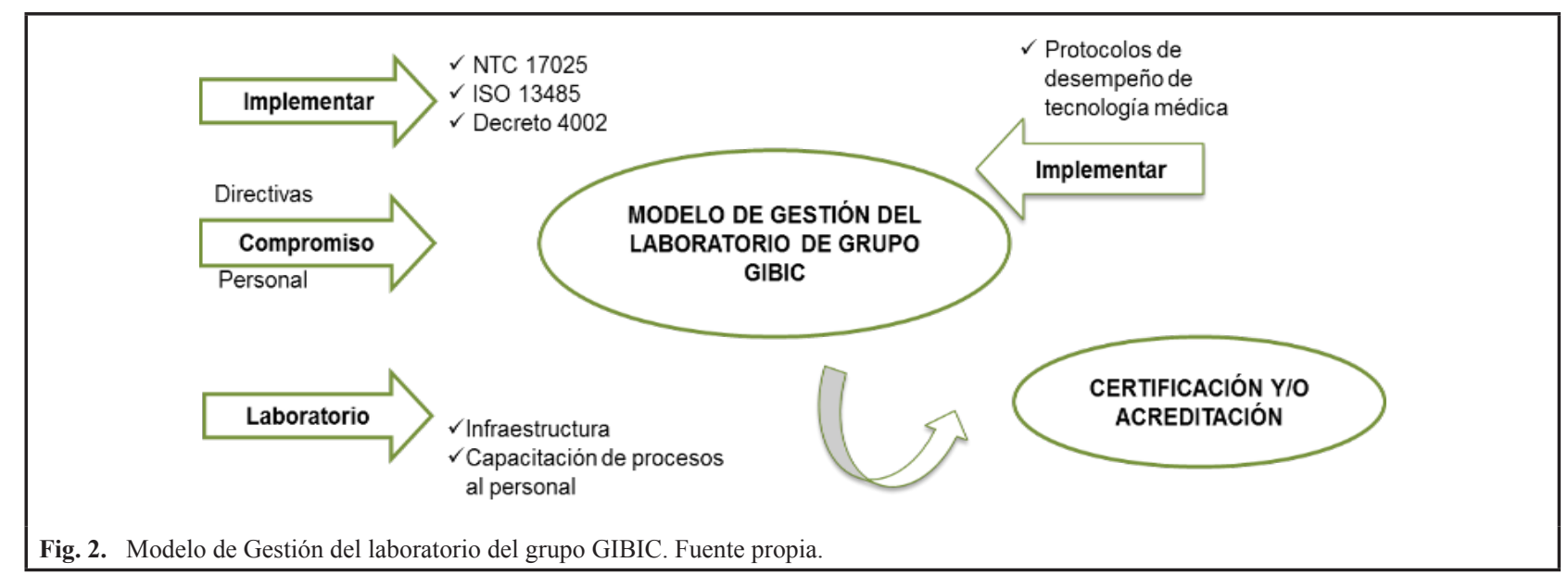




\section{Diseño y validación de los protocolos de desempeño necesarios para evaluación de tecnología médica en pre y posmercado dentro del alcance de la capacidad operativa del laboratorio}

Para el diseño de los protocolos de evaluación de tecnología médica se tuvo en cuenta la capacidad operativa del laboratorio, ya que según los equipos de evaluación con los que cuenta, se diseñaron el tipo de pruebas a realizar teniendo en cuenta el acceso a la documentación normativa que tiene la Universidad de Antioquia para referenciar dicho protocolo.

Se realizó un inventario de los equipos del GIBIC destinados a realizar las pruebas de desempeño y se definieron cuáles iban a ser utilizados en el laboratorio, especificando capacidad y magnitud de medida. A continuación, se enumeran los equipos de evaluación.

1. Analizador de desfibriladores

2. Analizador de electrobisturí

3. Analizador de flujo de gases

4. Analizador de seguridad eléctrica

5. Pozo seco de campo

6. Manómetro de precisión

7. Osciloscopio biomédico

8. Simulador de pulsoximetría

9. Simulador de signos vitales

10. Tacómetro

11. Termómetro
Para el diseño del protocolo se buscó que fuera general para cada tipo de tecnología e incluyera un número de pruebas para su evaluación. Posteriormente al diseño de protocolo, se validó en diferentes tipos de tecnología; inicialmente, se validó la seguridad eléctrica en una autoclave y una cámara hiperbárica de fabricantes nacionales de la ciudad de Medellín, las demás secciones del protocolo fueron validadas en un monitor de signos vitales comercial, un desfibrilador externo automático y en el prototipo de presión flexible, una innovación propia del GIBIC.

\section{Resultados}

\section{Modelos de gestión}

Para el desarrollo de esta etapa del proyecto, se realizó un estudio de modelos de gestión en que se analizaron los procesos realizados para la evaluación de desempeño de la tecnología médica propuestos por diferentes asociaciones gremiales, con el fin de brindar confiabilidad al paciente frente al uso de la tecnología médica. En la Tabla 1 se observa un comparativo de los modelos de gestión.

\section{Regulación}

En la Tabla 2, se pueden observar los diferentes entes reguladores a nivel mundial de la tecnología médica, la clasificación que cada uno le da a esta según el riesgo que tiene frente al paciente, donde I es riesgo leve, II moderado, III Y IV muy alto y el registro que otorgan para que esta pueda ser comercializada [8].

Tabla 1. Comparativo de modelos de gestión basado en [6] - [8]

\begin{tabular}{|c|c|c|}
\hline MODELO & VENTAJAS & DESVENTAJAS \\
\hline $\begin{array}{l}\text { AAMI } \\
\text { Association for } \\
\text { the Advancement of Medical } \\
\text { Instrumentation }\end{array}$ & $\begin{array}{l}\text { Modelo centrado al manejo del riesgo de los equipos. } \\
\text { Define los roles de los participantes del modelo, desde } \\
\text { la junta hasta los miembros del personal. }\end{array}$ & $\begin{array}{l}\text { No es explícito sobre indicadores de gestión } \\
\text { de la tecnología }\end{array}$ \\
\hline $\begin{array}{l}\text { ACCE } \\
\text { the American College of Clinical } \\
\text { Engineering }\end{array}$ & $\begin{array}{l}\text { Realza la labor del ingeniero clínico en la gestión } \\
\text { biomédica } \\
\text { Se discriminan actividades realizadas para una buena } \\
\text { adquisición de tecnología médica }\end{array}$ & $\begin{array}{l}\text { Considera solo la etapa de adquisición de } \\
\text { tecnología médica } \\
\text { No sugiere indicadores } \\
\text { Solo especifica funciones del ingeniero clínico }\end{array}$ \\
\hline $\begin{array}{l}\text { CENETEC } \\
\text { Centro Nacional de Excelencia } \\
\text { Tecnológica en Salud }\end{array}$ & $\begin{array}{l}\text { Guía el proceso de evaluación de tecnología médica } \\
\text { enfoque integral, abarcando aspectos éticos, clínicos y } \\
\text { económicos }\end{array}$ & $\begin{array}{l}\text { Orientado principalmente a la adquisición de } \\
\text { tecnología } \\
\text { No se encuentran definidos la asignación de } \\
\text { roles y responsabilidades de los participantes } \\
\text { del proceso }\end{array}$ \\
\hline $\begin{array}{l}\text { MHRA } \\
\text { the Medicines and Healthcare } \\
\text { Products Regulatory Agency }\end{array}$ & $\begin{array}{l}\text { Proceso detallado para cada una de las fases del modelo } \\
\text { incluye procesos de contaminación, dadas de bajas de } \\
\text { tecnología y disposición final } \\
\text { abarca a todos los participantes del proceso en la gestión }\end{array}$ & No especifica los indicadores de gestión \\
\hline $\begin{array}{l}\text { MPS } \\
\text { Ministerio de protección social }\end{array}$ & $\begin{array}{l}\text { Mejoramiento en la gestión de tecnología médica por } \\
\text { parte del personal asistencial }\end{array}$ & $\begin{array}{l}\text { Indicadores de mantenimientos y traslados de } \\
\text { tecnología }\end{array}$ \\
\hline
\end{tabular}


Tabla 2. Entes reguladores a nivel mundial [13]

\begin{tabular}{|c|c|c|c|c|c|c|}
\hline PAÍS & $\begin{array}{c}\text { ENTE } \\
\text { REGULADOR }\end{array}$ & I & II & III & IV & REGISTRO \\
\hline Argentina & ANMAT & $\mathrm{X}$ & $\mathrm{X}$ & $\mathrm{X}$ & & ANMAT \\
\hline Brasil & ANVISA & $\mathrm{X}$ & $\mathrm{X}$ & $\mathrm{X}$ & & ANVISA \\
\hline Colombia & INVIMA & $\mathrm{X}$ & $\begin{array}{l}\text { Ia riesgo moderado } \\
\text { /IIb riesgo Alto }\end{array}$ & $\mathrm{X}$ & $\mathrm{X}$ & REGISTRO SANITARIO \\
\hline Estados Unidos & FDA & $\mathrm{X}$ & $\mathrm{X}$ & $\mathrm{X}$ & & $510(\mathrm{k})$ \\
\hline México & COFEPRIS & $\mathrm{X}$ & $\mathrm{X}$ & $\mathrm{X}$ & & COFEPRIS \\
\hline Perú & DIGEMID & $\mathrm{X}$ & $\mathrm{X}$ & $\mathrm{X}$ & $\mathrm{X}$ & REGISTRO SANITARIO \\
\hline Unión Europea & $\begin{array}{l}\text { NBS (cuerpo } \\
\text { notificado) }\end{array}$ & $\mathrm{X}$ & $\mathrm{X}$ & $\mathrm{X}$ & & $\mathrm{CE}$ \\
\hline
\end{tabular}

\section{Protocolos de evaluación de desempeño}

A nivel mundial existen protocolos establecidos para determinar fiabilidad de la tecnología médica. En la Tabla 3 , se describen las principales características de los protocolos de evaluación de desempeño de tecnología médica.

Laboratorio de ingeniería clínica del GIBIC: estructura organizativa

Para definir la estructura organizativa del laboratorio del GIBIC, se tuvo en cuenta la capacidad de personal con el que esta cuenta para trabajar.

Definición de responsabilidades en el laboratorio de evaluación de desempeño de tecnología médica.

Auditor interno: es el responsable del funcionamiento del laboratorio ante la dirección, encargado de documentar todos los procesos realizados en este, recepción de tecnología, almacenamiento, etiquetado, pruebas de desempeño, y despacho de la tecnología médica.
Director técnico: encargado de vigilar los procesos realizados en el laboratorio, que se dé cumplimiento a las normas NTC-ISO-17025, NTC-ISO-13485 y la resolución 4002 [5]-[7]. Es el responsable por el laboratorio ante los entes de control.

Jefe de producción: este depende directamente del responsable de calidad, con funciones a cargo como: Planificar los recursos humanos, técnicos y administrativos de acuerdo con la dirección de calidad, es el responsable de la formación continua, en materia de ensayos, planificación y dirección de las auditorías internas y reuniones de revisión del sistema de calidad del laboratorio,

Personal operativo: es el encargado de realizar las funciones de clasificación de tecnología médica según las pruebas de desempeño a realizar, ejecutan las pruebas según las normas y procedimientos establecidos aplicando los protocolos.

Tabla 3. Protocolos de evaluación de desempeño de tecnología médica [8]-[10]

\begin{tabular}{|c|c|}
\hline ENTIDAD & CARACTERISTÍCA \\
\hline & Determina funcionalidad de esfigmomanómetros \\
\hline AAMI & Verificación de rangos establecidos \\
\hline & Verificación de rangos establecidos de esfigmomanómetros \\
\hline BHS & Establece máximos y mínimos basado en a OIML R16-1. \\
\hline ESH & $\begin{array}{l}\text { Añadió ciertos requisitos específicos para determinar la fiabilidad de los esfigmomanómetros en un grupo de } \\
\text { pacientes y que deben cumplirse para poder recomendar dichos dispositivos }\end{array}$ \\
\hline La Unión Europea & $\begin{array}{l}\text { EN 1060-Part IV. Exige la obtención del sello de calidad, un reconocido galardón que cuenta con la homologación } \\
\text { de la Liga Alemana contra la Hipertensión. La base para la prueba clínica es el DIN EN 540, ampliado con más } \\
\text { requisitos relacionados, por ejemplo, con la selección del observador y la evaluación de los resultados. }\end{array}$ \\
\hline $\begin{array}{l}\text { Fluke corporación, Universidad } \\
\text { de Vermot }\end{array}$ & $\begin{array}{l}\text { 1. Protocolos de pruebas de desempeño basados en: Mantenimientos preventivos } \\
\text { 2. Inspección interna } \\
\text { 3. Normalización (IEC60601) }\end{array}$ \\
\hline
\end{tabular}


Estructura documental: las actividades a realizar en el laboratorio son las de evaluar el desempeño, la gestión de calidad y el almacenamiento y acondicionamiento de la tecnología médica, por lo tanto se respetan los siguientes lineamientos de la NTC-ISO 17025, NTC-ISO 13485 y la resolución 4002[5]-[7]. Los cuales se encuentran totalmente documentados.

Requisitos de gestión: revisión de los pedidos, ofertas y contratos. Cuando un cliente solicita las pruebas dentro del alcance del laboratorio, está implícito que los informes/certificados emitidos estén soportados por la documentación del personal técnico que los realiza, para así demostrar la idoneidad de personal.

Control de los registros: los registros se conservan al menos, durante 5 años o el periodo que establezcan otras disposiciones legales aplicables.

Auditorías internas: se llevarán a cabo una vez al semestre y se programarán auditorías de revisión de acuerdo con los hallazgos reportados.

Revisiones por la dirección: el sistema de calidad adoptado por el laboratorio será revisado, una vez al semestre en el primer año, y posterior a esto una vez al año.

Planificación del diseño y desarrollo: el laboratorio planifica y controla el diseño y desarrollo del producto, las validaciones apropiadas para cada una de sus etapas, también establece procedimientos documentados.

Resultados del diseño y desarrollo (tecnología en premercado): los resultados del diseño y desarrollo se proporcionan de tal manera que permitan la verificación respecto a los elementos de entrada para el diseño y desarrollo, y se aprueban antes de su liberación.

Verificación del diseño y desarrollo (tecnología en premercado): se realiza la verificación, de acuerdo con lo planificado para asegurarse de que los resultados del diseño y desarrollo cumplen los requisitos de los elementos de entrada del diseño y desarrollo. Se mantienen los registros de resultados de la verificación y de cualquier acción que sea necesaria.

Requisitos específicos para el almacenamiento y acondicionamiento de tecnología médica (tecnología en pre y posmercado)

\section{Aspectos generales}

Se realiza la inspección inicial de la tecnología médica e insumos en el momento de su recepción y se deja la evidencia documental correspondiente en un formato establecido como lista de chequeo. La tecnología médica cuenta con los respectivos certificados de calidad o certificados de conformidad proporcionados por los fabricantes, los cuales se encuentran disponibles en archivos físicos o medios electrónicos.

\section{Instalaciones}

Por medio de un recorrido realizado en el laboratorio $\mathrm{y}$, verificando los lineamientos exigidos por las normas aplicadas, se evidenció que las instalaciones son apropiadas para las operaciones que se realizan en ellas, ya que su ubicación y funcionamiento no generan riesgo para la salud de las personas que habitan en edificaciones circundantes o aledañas.

\section{Condiciones externas}

Se realizaron algunos cambios correctivos como recuperación de grietas y revoques en las paredes y baldosas, para que las edificaciones no tuvieran algún deterioro que pudiera afectar a la tecnología.

\section{Condiciones internas}

Se verificó que las paredes, pisos y techos de las áreas donde se realizan actividades no tuvieran grietas ni humedades.

\section{Zonas y áreas específicas del laboratorio}

\section{Recepción (tecnología en pre y posmercado)}

Esta cuenta con suficiente espacio y elementos necesarios para permitir la revisión previa de la tecnología médica antes de su ingreso al laboratorio.

\section{Zona de etiquetado (tecnología en premercado)}

Dispone de una zona definida, con espacio suficiente y cuenta con personal capacitado para el desarrollo las actividades, los materiales y elementos.

\section{Zona de empaque (tecnología en premercado)}

El laboratorio cuenta con una zona para el alistamiento de la tecnología médica acondicionada para su empaque secundario o embalaje final.

\section{4. Área de almacenamiento (tecnología en pre y posmercado)}

El laboratorio cuenta con áreas de almacenamiento divididas (para tecnología médica aprobada para el despacho, demostraciones, capacitación a usuarios, en cuarentena y tecnología retirada y devuelta o rechazada), dispuestas tanto física como funcionalmente. De acuerdo con el tipo de tecnología médica almacenada se ubica de forma independiente, en el caso de que el fabricante lo recomiende.

\section{5. Área de Pruebas de desempeño (tecnología en pre} y posmercado)

Se dispuso de una zona definida, con espacio y dotación suficiente, la cual cuenta con personal capacitado 
y equipos para desarrollar las pruebas de desempeño. Los equipos que se utilizarán en esta área son totalmente trazables y tienen la periodicidad de actividades de mantenimiento preventivo y calibración correctamente. A continuación, en la Fig. 3 se muestra una de las zonas esta área.

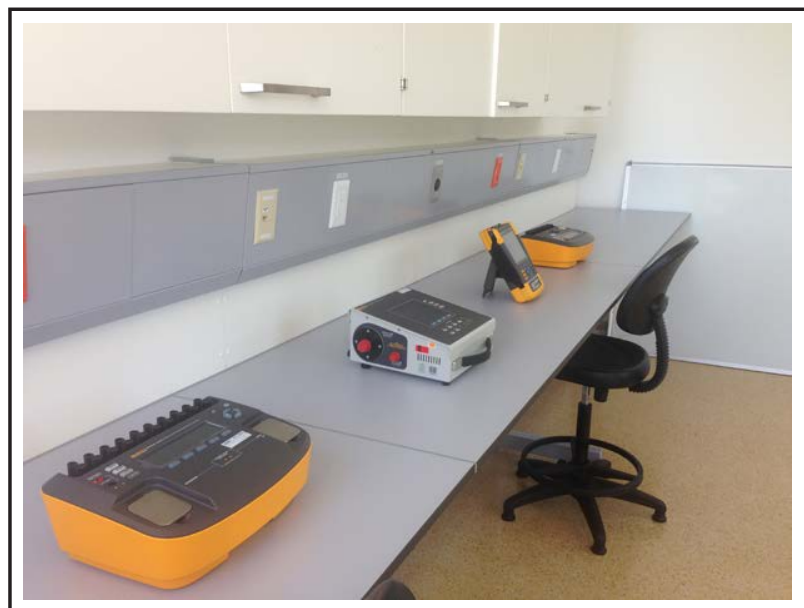

Fig. 3 Área de pruebas de desempeño laboratorio del GIBIC

\section{Capacidad operativa}

Los equipos para evaluación de las pruebas de desempeño de la tecnología médica tienen una adecuada trazabilidad de actividades de mantenimiento preventivo y calibración, con documentación soportada para realizar las evaluaciones de magnitudes y seguridad eléctrica. En la Tabla 4 se evidencian los equipos para evaluación con los que cuenta el laboratorio.

Tabla 4. Equipos para evaluación

\begin{tabular}{|l|l|l|}
\hline Analizador de desfibriladores & & Osciloscopio biomédico \\
Analizador de electrobisturí & & Simulador de pulsoximetría \\
\hline Analizador de flujo de gases & & Simulador de signos vitales \\
\hline Analizador de seguridad elétrica & & Tacómetro Amprobe \\
\hline Bloque seco de temperatura & Termómetro \\
&
\end{tabular}

\section{Modelo de gestión del laboratorio de ingeniería clíni- ca del GIBIC}

El modelo de gestión del laboratorio da cumplimiento a los estándares de calidad y a los requisitos mínimos para alcanzar una certificación en la resolución 4002 del Ministerio de Protección Social, por la cual se adopta el manual de requisitos de Capacidad de Almacenamiento y/o Acondicionamiento para Dispositivos Médicos. Este proceso demuestra de una forma efectiva las competencias técnicas, dando credibilidad de los servicios que ofrece. Para alcanzar dicha certificación se debe tener la documentación del sistema de gestión de calidad, especificando la estructura organizativa, documental y de la capacidad del laboratorio; también es necesario documentar el protocolo para la evaluación de desempeño de la tecnología médica con sus procedimientos de ensayo, donde se describa la secuencia de operaciones y métodos aplicados, incluyendo la trazabilidad de los equipos utilizados para estas evaluaciones y las capacitaciones realizadas sobre los procesos al personal del laboratorio.

\section{Protocolo de pruebas de evaluación de desempeño}

El protocolo de pruebas de evaluación de desempeño de tecnología médica se realizó basado en la capacidad del laboratorio, teniendo en cuenta normatividades para evaluar este tipo de tecnología, las secciones que se encuentran en el protocolo son las siguientes:

1. Identificación de partes y accesorios: se realiza una inspección visual del equipo. Se verifica el estado y la operatividad según las especificaciones dadas por el fabricante [4][10].

2. Pruebas de seguridad eléctrica (requisitos generales IEC-60601): se realizan con el fin de garantizar el buen funcionamiento de la tecnología médica y para asegurar la protección del paciente, debido a que un accidente eléctrico con un equipo médico puede dañar tejidos $u$ órganos. Las medidas de seguridad eléctricas para estos están enfocadas en prevenir la fibrilación y otras anomalías menores. Las normas de seguridad eléctrica requieren que las corrientes que puedan tener contacto con el paciente sean mínimas. La IEC-60601 es la norma de obligatorio cumplimiento para garantizar la seguridad eléctrica de los equipos médicos [11].

3. Identificación de magnitudes: para estas pruebas se define la resolución y rango de medición con el que trabaja la tecnología. El análisis de las magnitudes se hace con un equipo de referencia totalmente trazable, estableciendo los puntos críticos según el fabricante [4].

4. Pruebas de software según la norma IEC 62304: en estas se tiene en cuenta que el software a analizar no es independiente y se encuentra incorporado en la tecnología, por lo que se evalúa el comportamiento de las variables de entrada y salida y los rangos establecidos por el fabricante [12].

5. Pruebas de usabilidad IEC 62366-1: con estas, se verifica que el equipo sea fácil de usar para el personal que lo va a utilizar. Se realiza, posteriormente, a una capitación de manejo del equipo, tomando como referencia el tiempo utilizado por la persona experta en la operatividad del equipo [13]. 
6. Pruebas de alarmas según la IEC 60601-1-8: con estas se verifican alarmas visuales y auditivas de la tecnología que indiquen mal funcionamiento o parámetros fuera del rango programado [14].

7. Pruebas de carcasa según la norma MIL-STD-810: estas son específicas para prototipos, es decir, tecnologías que aún no han empezado su periodo de comercialización.

\section{Validación de protocolo de pruebas de desempeño}

El protocolo de evaluación de desempeño de tecnología médica fue validado en diferentes tipos de tecnología. Inicialmente, se validó la seguridad eléctrica en una autoclave y una cámara hiperbárica de productores nacionales donde se realizaron mediciones de: fluctuaciones de voltaje, prueba límite de tensión y pruebas de corriente de fuga.

La validación de la sección de seguridad eléctrica se realizó con mediciones de voltaje de la red de alimentación con respecto al conductor simple (tierra), teniendo como valor nominal $110 \mathrm{~V} \pm 10 \%$. Se realizaron las mediciones de impedancia entre conexión de clavija del toma corriente y parte metálica del equipo accesible, comparando el valor obtenido con el rango permitido que es $<0,2 \Omega$.

\section{Pruebas de seguridad eléctrica autoclave}

Se obtuvo un voltaje de 119,5 V; 9,5 > Valor nominal, el cual está entre el rango permitido. Se realizó la medición de la impedancia entre conexión de clavija del toma corriente y parte metálica del equipo accesible y se obtuvo una impedancia de $0,014 \Omega$ menor al valor permitido que es $<0,2 \Omega$, las demás mediciones de corriente de fuga dentro del rango establecido, lo cual muestra el cumplimiento de seguridad eléctrica.

\section{Pruebas de seguridad eléctrica cámara hiperbárica}

Se obtuvo un voltaje de $115,5 \mathrm{~V} ; 5,5 \mathrm{~V}>$ Valor nominal, el cual está entre el rango permitido, en la prueba límite de tensión se obtuvo una impedancia $0,038 \Omega<0,2$ $\Omega$ al valor permitido, lo cual muestra el cumplimiento con el límite establecido.

Para la medida de corriente de fuga de conductor, se realizaron las pruebas de:

- Fuga de conductor simple (Fuga de chasis): es la medida del flujo de corriente desde el chasis del equipo a través de la carga a tierra de la fuente de alimentación. Esta prueba se hizo con las opciones de polaridad inversa, tierra abierta y neutro abierta.

Tabla 5. Seguridad eléctrica autoclave

FLUCTUACIONES DE VOLTAJE

\begin{tabular}{c|c|c|c|c}
\hline Variable & Rango permitido & Valor nominal & Valor medido & Pasa/No pasa \\
\hline Voltaje & $\pm 10 \%$ del valor nominal $(\mathrm{V})$ & 110 & 119,5 & Pasa \\
\hline
\end{tabular}

PRUEBA DE LIMITACIÓN DE TENSIÓN

\begin{tabular}{|c|c|c|c|c|}
\hline Variable & Rango permitido & Medida ( $\Omega)$ & Pasa & No Pasa \\
\hline Impedancia & $<0,2 \Omega$ & 0,01414 & $\mathrm{X}$ & \\
\hline
\end{tabular}

PRUEBAS DE CORRIENTE DE FUGA

\begin{tabular}{|c|c|c|c|c|}
\hline \multicolumn{2}{|c|}{ Condición } & \multirow{2}{*}{$\begin{array}{c}\text { Límite establecido } \\
\qquad(\boldsymbol{\mu A})\end{array}$} & \multirow{2}{*}{$\begin{array}{c}\text { Medida }(\boldsymbol{\mu A}) \\
88\end{array}$} & \multirow{2}{*}{$\begin{array}{l}\text { Pasa } \\
\\
\mathrm{X}\end{array}$} \\
\hline \multirow{2}{*}{ Corriente de fuga a tierra } & $\begin{array}{l}\text { Condición primer defecto neutro } \\
\text { abierto }\end{array}$ & & & \\
\hline & $\begin{array}{c}\text { Condición primer defecto polaridad } \\
\text { invertida }\end{array}$ & 1000 & 202 & $\mathrm{X}$ \\
\hline Corriente de fuga a carcasa & Condición normal & 500 & 259 & $\mathrm{X}$ \\
\hline \multirow{3}{*}{ Corriente de fuga a carcasa } & Condición primer defecto tierra abierta & 500 & 259 & $\mathrm{X}$ \\
\hline & $\begin{array}{l}\text { Condición primer defecto neutro } \\
\text { abierto }\end{array}$ & 500 & 259 & $\mathrm{X}$ \\
\hline & $\begin{array}{c}\text { Condición primer defecto polaridad } \\
\text { invertida }\end{array}$ & 500 & 259 & $\mathrm{X}$ \\
\hline
\end{tabular}


Tabla 6. Seguridad eléctrica de cámara hiperbárica

\section{FLUCTUACIONES DE VOLTAJE}

\begin{tabular}{c|c|c|c|c}
\hline Variable & Rango permitido & Valor nominal & Valor medido & Pasa/No pasa \\
\hline Voltaje & $\pm 10 \%$ del valor nominal $(\mathrm{V})$ & $110 \mathrm{~V}$ & \multicolumn{3}{c}{$115,5 \mathrm{~V}$} & Pasa \\
\hline \multicolumn{5}{c|}{ PRUEBA DE LIMITACIÓN DE TENSIÓN } \\
\hline Variable & Rango permitido & Medida $(\Omega)$ & Pasa & No Pasa \\
\hline Impedancia & $<0,2 \Omega$ & $0,038 \Omega$ & $\mathrm{X}$ & \\
\hline
\end{tabular}

PRUEBAS DE CORRIENTE DE FUGA

\begin{tabular}{|c|c|c|c|c|}
\hline \multicolumn{2}{|r|}{ Condición } & Límite establecido $(\mu \mathrm{A})$ & Medida $(\mu \mathrm{A})$ & Pasa \\
\hline Corriente de fuga a tierra & Condición primer defecto neutro abierto & 1000 & 0,7 & $\mathrm{X}$ \\
\hline \multirow{2}{*}{ Corriente de fuga a tierra } & Condición primer defecto polaridad invertida & 1000 & 58 & $\mathrm{X}$ \\
\hline & Condición normal & 500 & 63 & $\mathrm{X}$ \\
\hline \multirow{2}{*}{ Corriente de fuga a carcasa } & Condición primer defecto tierra abierta & 500 & 0,5 & $\mathrm{X}$ \\
\hline & Condición primer defecto neutro abierto & 500 & 0,4 & $\mathrm{X}$ \\
\hline Corriente de fuga a carcasa & Condición primer defecto polaridad invertida & 500 & 0,4 & $\mathrm{X}$ \\
\hline
\end{tabular}

Las secciones del protocolo en cuanto a identificación de partes y/o accesorios, pruebas de magnitudes y pruebas de software se validaron en las siguientes tecnologías.

Monitor de Signos Vitales. Secciones de identificación de partes $\mathrm{y} / \mathrm{o}$ accesorios, pruebas de magnitudes y pruebas de software.

Tabla 7. Identificación de partes y/o accesorios

\begin{tabular}{cc}
\hline inspección visual & Bueno \\
\hline Alarmas & $\mathrm{X}$ \\
Batería & $\mathrm{X}$ \\
Brazalete & $\mathrm{X}$ \\
Cable de ECG & $\mathrm{X}$ \\
Sensor de SPO2 & $\mathrm{X}$ \\
Botón de encendido/ Apagado & $\mathrm{X}$ \\
\hline
\end{tabular}

Tabla 8. Pruebas de presión no invasiva

\begin{tabular}{|c|c|c|c|c|c|c|}
\hline \multicolumn{7}{|c|}{ Medición de presión no invasiva (NIBP) } \\
\hline \multirow[b]{2}{*}{ Presiones } & \multirow{2}{*}{$\begin{array}{c}\text { Valor de } \\
\text { referencia } \\
\text { mmHg }\end{array}$} & \multicolumn{3}{|c|}{ Lectura de Medición } & \multirow{2}{*}{$\begin{array}{l}\text { Promedio } \\
\text { de lecturas }\end{array}$} & \multirow[b]{2}{*}{ Error } \\
\hline & & $\begin{array}{c}\text { Lectura } \\
1\end{array}$ & $\begin{array}{c}\text { Lectura } \\
2\end{array}$ & $\begin{array}{c}\text { Lectura } \\
3\end{array}$ & & \\
\hline Sistólica & 90 & 90 & 90 & 91,9 & 90,6 & 0,6 \\
\hline Diastólica & 60 & 60 & 60 & 60 & 60 & 0 \\
\hline Sistólica & 120 & 120,2 & 120 & 120 & 120,1 & 0,1 \\
\hline Diastólica & 90 & 89,5 & 90 & 90 & 89,8 & 0,2 \\
\hline Sistólica & 150 & 149,5 & 150 & 150 & 149,8 & 0,2 \\
\hline Diastólica & 110 & 110 & 110 & 110 & 110 & 0 \\
\hline Sistólica & 180 & 178,6 & 180 & 180 & 179,5 & 0,5 \\
\hline Diastólica & 120 & 120 & 120 & 120 & 120 & 0 \\
\hline Sistólica & 200 & 200 & 200 & 200 & 200 & 0 \\
\hline Diastólica & 160 & 160 & 160 & 160 & 160 & 0 \\
\hline
\end{tabular}

Tabla 9. Pruebas de frecuencia cardíaca

\begin{tabular}{|c|c|c|c|c|c|c|}
\hline \multicolumn{7}{|c|}{ Medición de frecuencia cardíaca (EKG) } \\
\hline \multirow{2}{*}{$\mathbf{n}$} & \multirow{2}{*}{$\begin{array}{c}\text { Valor de } \\
\text { referencia } \\
\text { [bpm] }\end{array}$} & \multicolumn{3}{|c|}{ Lectura de Medición } & \multirow{2}{*}{$\begin{array}{c}\text { Promedio de } \\
\text { lecturas }\end{array}$} & \multirow{2}{*}{ Error } \\
\hline & & $\begin{array}{c}\text { Lectura } \\
1\end{array}$ & \begin{tabular}{|c|} 
Lectura \\
2
\end{tabular} & \begin{tabular}{|c|} 
Lectura \\
3
\end{tabular} & & \\
\hline 1 & 20 & 20 & 20 & 20 & 20 & 0 \\
\hline 2 & 40 & 40 & 40 & 40 & 40 & 0 \\
\hline 3 & 60 & 60 & 60 & 60 & 60 & 0 \\
\hline 4 & 80 & 80 & 80 & 80 & 80 & 0 \\
\hline 5 & 100 & 100 & 100 & 100 & 100 & 0 \\
\hline 6 & 120 & 120 & 120 & 120 & 120 & 0 \\
\hline 7 & 140 & 140 & 140 & 140 & 140 & 0 \\
\hline 8 & 160 & 160 & 160 & 160 & 160 & 0 \\
\hline 9 & 180 & 180 & 180 & 180 & 180 & 0 \\
\hline 10 & 200 & 200 & 200 & 200 & 200 & 0 \\
\hline
\end{tabular}

Tabla 10. Pruebas de software

\begin{tabular}{lc}
\hline \multicolumn{1}{c}{ Funcionamiento } & Bueno \\
\hline Abren correctamente y presentan lo indicado & $\mathrm{X}$ \\
Realizan correctamente el registro y el cierre & $\mathrm{X}$ \\
Es necesario datos de autenticidad & $\mathrm{X}$ \\
$\begin{array}{l}\text { Discriminación de tipo de letra en contraseñas } \\
\text { Se puede regresar al menú principal luego }\end{array}$ & $\mathrm{X}$ \\
después de seleccionado una opción & $\mathrm{X}$ \\
Almacenamiento de datos ingresados & $\mathrm{X}$ \\
Registro de información & $\mathrm{X}$ \\
\hline
\end{tabular}

Monitor de signos vitales la presión no invasiva se obtuvo un error máximo de $0,6 \mathrm{mmHg}<3 \mathrm{mmHg}$ EMP; para la frecuencia cardíaca, se obtuvo un error de 0,0 bpm $<$ 1 bpm EMP y esto indicó que el equipo estaba funcionando 
en los valores adecuados descritos por el fabricante en cuanto a sus parámetros de presión y frecuencia cardíaca. Los parámetros evaluados en las pruebas de software fueron totalmente correctos en cuanto al funcionamiento del software externo del monitor de signos vitales.

Desfibrilador Externo Automático (DEA). Secciones de identificación de partes y/o accesorios, pruebas de magnitudes, pruebas de software

Tabla 11. Identificación de partes y/o accesorios

\begin{tabular}{|c|c|c|c|c|c|c|}
\hline \multirow{2}{*}{$\mathbf{n}$} & \multirow{2}{*}{$\begin{array}{c}\text { Valor de } \\
\text { referencia } \\
{[J](50 \Omega)}\end{array}$} & \multicolumn{2}{|c|}{$\begin{array}{l}\text { Lectura de } \\
\text { Medición }\end{array}$} & \multirow{2}{*}{ Promedio } & \multirow{2}{*}{ Error } & \multirow{2}{*}{$\begin{array}{c}\% \\
\text { Error }\end{array}$} \\
\hline & & $\begin{array}{c}\text { Lectura } \\
1 \\
\end{array}$ & $\begin{array}{c}\text { Lectura } \\
2\end{array}$ & & & \\
\hline 1 & 120 & 117 & 117 & 117 & 3 & 2,5 \\
\hline 2 & 180 & 180 & 180 & 180 & 0 & 0 \\
\hline 3 & 240 & 249 & 249,9 & 249,5 & 9,5 & 4,0 \\
\hline 4 & 270 & 270 & 270 & 270 & 0 & 0 \\
\hline
\end{tabular}

Tabla 12. Pruebas de energía

\begin{tabular}{lc}
\hline \multicolumn{1}{c}{ Inspección visual } & Bueno \\
\hline Parches & $\mathrm{X}$ \\
Batería & $\mathrm{X}$ \\
Botón de selección de energía & $\mathrm{X}$ \\
Chasis del equipo & $\mathrm{X}$ \\
Enchufe de red y base de enchufe & $\mathrm{X}$ \\
\hline
\end{tabular}

Tabla 13. Pruebas de frecuencia cardíaca

\begin{tabular}{|c|c|c|c|c|c|c|}
\hline \multicolumn{7}{|c|}{ Medición de frecuencia cardíaca (ECG) } \\
\hline \multirow[b]{2}{*}{$\mathbf{n}$} & \multirow{2}{*}{$\begin{array}{c}\text { Valor de } \\
\text { referencia } \\
\text { [bpm] }\end{array}$} & \multicolumn{3}{|c|}{ Lectura de Medición } & \multirow{2}{*}{$\begin{array}{c}\text { Promedio } \\
\text { de lecturas }\end{array}$} & \multirow[b]{2}{*}{ Error } \\
\hline & & $\begin{array}{c}\text { Lectura } \\
1\end{array}$ & $\begin{array}{c}\text { Lectura } \\
2\end{array}$ & $\begin{array}{c}\text { Lectura } \\
3\end{array}$ & & \\
\hline 1 & 20 & 20 & 20 & 20 & 20 & 0 \\
\hline 2 & 40 & 40 & 40 & 40 & 40 & 0 \\
\hline 3 & 60 & 60 & 60 & 60 & 60 & 0 \\
\hline 4 & 80 & 80 & 80 & 80 & 80 & 0 \\
\hline 5 & 100 & 100 & 100 & 100 & 100 & 0 \\
\hline 6 & 120 & 120 & 120 & 120 & 120 & 0 \\
\hline 7 & 140 & 140 & 140 & 140 & 140 & 0 \\
\hline 8 & 160 & 160 & 160 & 160 & 160 & 0 \\
\hline 9 & 180 & 180 & 180 & 180 & 180 & 0 \\
\hline 10 & 200 & 200 & 200 & 200 & 200 & 0 \\
\hline
\end{tabular}

Tabla 14. Pruebas de software

\begin{tabular}{lc}
\hline \multicolumn{1}{c}{ Funcionamiento } & Bueno \\
\hline Abren correctamente y presentan lo indicado & $\mathrm{X}$ \\
Realizan correctamente el registro y el cierre & $\mathrm{X}$ \\
Es necesario datos de autenticidad & $\mathrm{X}$ \\
Discriminación de tipo de letra en contraseñas & $\mathrm{X}$
\end{tabular}

Se puede regresar al menú principal luego después de seleccionado una opción

Almacenamiento de datos ingresados

Registro de información
$\mathrm{X}$

$\mathrm{X}$

$\mathrm{X}$
Para el DEA (Desfibrilador externo automático) la evaluación de desempeño en partes y accesorios fue satisfactoria. Las magnitudes evaluadas de energía y frecuencia cardíaca estuvieron en el rango permitido por el fabricante, ya que no superó el EMP de energía que es el $10 \%$ y se obtuvo un error de $0,04 \%$, para la frecuencia cardíaca se obtuvo error de $0,0 \%<1 \%$ EMP y esto indicó que el esquipo estaba funcionando en los valores adecuados descritos por el fabricante, en cuanto a sus parámetros de energía y frecuencia cardíaca. Los parámetros evaluados en las pruebas de software fueron totalmente correctos, en cuanto al funcionamiento del software externo del desfibrilador externo automático (DEA).

Posteriormente de la validación del protocolo por secciones en diferentes tipos de tecnología, se realizaron las pruebas de desempeño en el prototipo de presión flexible del GIBIC.

\section{Prototipo de presión flexible del GIBIC}

Se contó con 4 prototipos de presión flexible, denominados llamadas IPS_1, IPS_2, IPS_3, IPS 4. La inspección visual de los 4 mantas de presión flexible en cuanto a partes y accesorios fue satisfactoria. En las pruebas de seguridad eléctrica, en la sección de fluctuación de voltaje para cada una de ellas, las mediciones fueron satisfactorias, ya que se evidenció que estaban en el rango permitido, no sobrepasaron el valor permitido con relación al valor nominal. La medición de la impedancia entre conexión de clavija del toma corriente y parte metálica del quipo accesible para, IPS_3 estaba en el límite permitido pero la para IPS_1, IPS_2 e IPS_4 sobrepasaron el límite, ya que los valores medidos son mayores a $0,2 \Omega$, del valor permitido. Las mediciones de corrientes de fuga fueron aceptables, ay que no sobrepasaron el límite establecido.

El prototipo de presión flexible se divide en 5 regiones. En la Fig. 4 se observan las regiones y en la Fig. 5 la visualización de la activación de los sensores del prototipo de presión flexible.

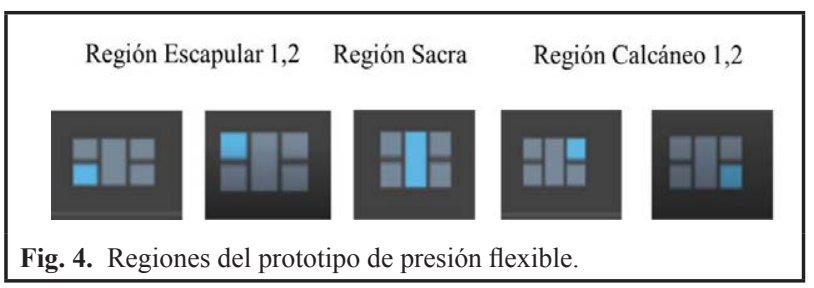




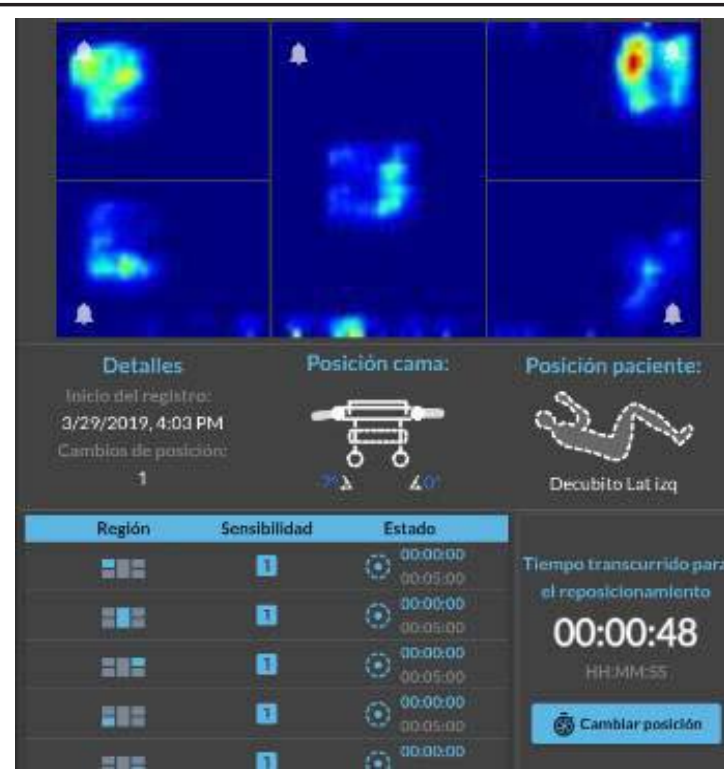

Fig. 5. Activación de sensores

Para realizar la prueba de presión, se aplicó una presión constante con cajas de maderas realizadas en el laboratorio de un peso constante de $5 \mathrm{Kg}$, esta presión se ejerció en todas las regiones de los prototipos para poder observar el funcionamiento del software y la activación de los senso- res. Por medio de la visualización en el software se logró ver la activación de los sensores de presión (Fig. 6).

Para IPS_1 e IPS_3 se observó la eficiencia de los sensores en las 5 regiones, además, con el software se detectó la presión ejercida. Para el prototipo IPS_2 no se detectó ninguna conectividad entre la manta y el software y, para IPS_3, la detección de la presión en la región calcáneo fue muy débil.

Las pruebas de software fueron satisfactorias para las variables de entrada y salida. Con las pruebas de usabilidad se comprobó que el software es de fácil entendimiento para el operador.

\section{Prueba de sensibilidad}

La sensibilidad es generada por la activación de los sensores que componen cada región, donde 1 es menor sensibilidad y 5 mayor sensibilidad. Para esta prueba se ejerció una presión igual en cada una de las regiones de la manta por cada nivel.

El comportamiento de la alerta, es decir, de la activación de esta en el software de acuerdo con la sensibilidad tuvo un comportamiento estable para la IPS_1, IPS 3 e IPS 4. En la región calcáneo se activó la alarma para todas las sensibilidades, en la región escapular se activó la alarma para las sensibilidades 2, 3, 4 y en la región sacra para las sensibilidades $3,4,5$.

\section{IPS_1}

Región Escapular 1,2
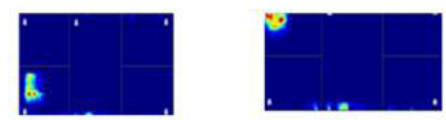

Región Calcáneo 1,2
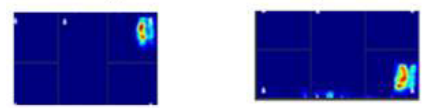

IPS_3

Región Escapular 1,2
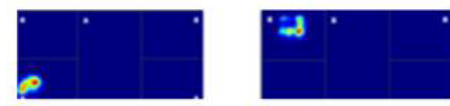

Región Sacra

Región Calcáneo 1,2
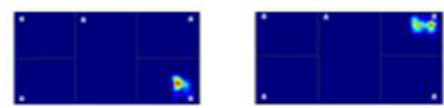

Región Sacra
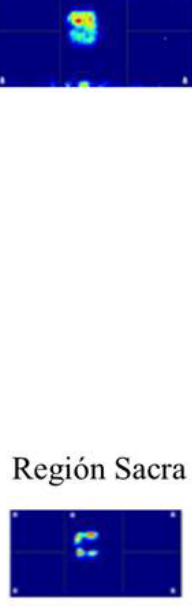

Fig. 6. Activación de sensores
IPS_2

Región Escapular 1,2
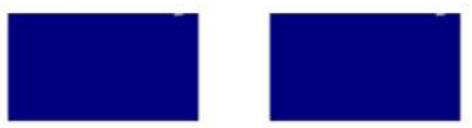

Región Sacra

Región Calcáneo 1,2
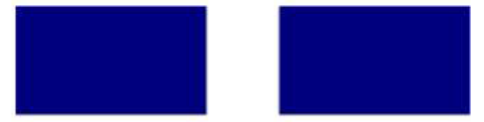

IPS_4
Región Escapular 1,2
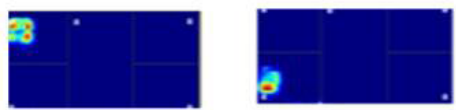

Región Calcáneo 1,2

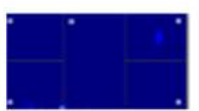

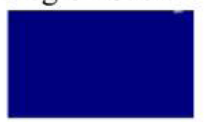

Región Sacra

z

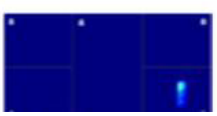




\section{Discusión}

Tener conocimiento de los entes reguladores de dispositivos médicos a nivel mundial facilita, identificar el nivel del riesgo que tiene cada tipo de tecnología médica frente al paciente y así poder establecer los lineamientos necesarios para validar su funcionamiento. De los modelos de gestión de evaluación de tecnología médica descritos, es importante poder identificar la necesidad de soportar la confiabilidad de funcionamiento de este tipo de tecnología. Los modelos MHRA, AAMI y MPS presentan un enfoque detallado en cuanto a la gestión de la tecnología médica y los ACCE y CENETEC le dan más importancia a la parte de adquisición. Por otra parte, los modelos anteriormente descritos se basan en el proceso de evaluación de funcionamiento clínico y adquisición, pero no se videncia un modelo de gestión para evaluar el desempeño de la tecnología médica en etapa de pre y posmercado, donde se evidencien las normativas que rigen a los laboratorios de ensayo, acondicionamiento, almacenamiento, calibración, y la gestión de calidad de tecnología médica, como la ONAC Organismo Nacional de Acreditación Colombia [3].

La mayoría de los protocolos comparados son protocolos del mismo tipo de tecnología donde, en cada uno de ellos, se logra evidenciar mejoras en las especificaciones técnicas. También se presentan protocolos basados en normas estandarizadas de seguridad eléctrica y verificación de especificaciones recomendadas por el fabricante. Este tipo de protocolos son variables dependiendo de la característica específica y funcionamiento por cada uno de los tipos de tecnología médica existente. En la tecnología evaluada, se evidencia esta variación en las secciones de partes y/o accesorios, identificación de magnitudes y usabilidad.

Mediante el estudio físico, operativo y de capacidad del laboratorio del GIBIC de la Universidad de Antioquia se logró diseñar un modelo de gestión para este, bajo normatividades necesarias para realizar pruebas de desempeño de tecnología médica en etapa de pre y posmercado. Por medio de las validaciones del protocolo realizadas a diferentes tipos de tecnología se pudo evidenciar que este contiene las secciones necesarias para realizar una correcta evaluación y verificar el cumplimiento de normatividades de seguridad eléctrica, de software, de usabilidad, estado físico y recomendaciones del fabricante. Los resultados de evaluaciones de desempeño se presentan mediante un informe de conformidad de funcionamiento de tecnología médica, especificando pruebas y evaluaciones que fueron realizadas. Este informe va a nombre del laboratorio del GIBIC.

Con los procesos realizados en el laboratorio del GIBIC se busca alcanzar la normalización y estandarización de validación de tecnología biomédica en etapa de pre y posmercado dando cumplimiento al capítulo III del decreto 4725 de 2005 que establece que todo fabricante y/o importador debe certificarse en buenas prácticas de manufactura, capacidad de almacenamiento y/o acondicionamiento de los dispositivos médicos.

\section{Conclusiones}

En Colombia existen laboratorios de validación de tecnología médica, también empresas reconocidas por prestar servicios de interventoría y control de calidad. Estos laboratorios realizan procesos de validación de acuerdo NTC/ ISO/IEC 17025:2017. Apoyados de procesos internos, pero son determinados para cada tipo de tecnología a validar, específicamente, en el caso de autoclaves, neveras y cadena de frío, pero no se cuenta con protocolos generales aplicables a una variedad de dispositivos como monitores de signos vitales, cámaras hiperbáricas, Desfibriladores, ventiladores mecánicos.

Por lo que el modelo de gestión del laboratorio para evaluación de desempeño de tecnología médica puede ser replicado por universidades que cuentan con laboratorios con la dotación necesaria y el personal capacitado, generando un gran beneficio para productores nacionales, ya que pueden validar sus tecnologías en un solo laboratorio que contiene un proceso protocolario de evaluación y expide un informe dando cumplimiento a los lineamientos del ente de vigilancia colombiano INVIMA.

\section{Agradecimientos}

A la Universidad de Antioquia, el grupo de Investigación GIBIC. Este trabajo ha sido financiado por Sistema General de Regalías, Republica de Colombia a través del proyecto "Fortalecimiento de la plataforma tecnológica para la formación especializada en el área de la salud y el desarrollo de tecnología biomédica", código RutaN-139C.

Los autores agradecen de manera especial a todas las instituciones prestadoras de servicios de salud a la empresa Calmet las Américas, las cuales me permitieron la manipulación de la tecnología médica para validar los protocolos.

\section{REFERENCIAS}

[1]. Cubillos M. C., Rozo, D. El concepto de calidad: historia, evolución e importancia para la competitividad. Revista de la Universidad de la Salle, 48, 80-99, 2009

[2]. Fontalvo, T. J., De La Hoz, E. J. Diseño e implementación de un sistema de gestión de la calidad ISO 9001: 2015 en una Universidad Colombiana. Formación universitaria, 11(1), 35-44, 2018 
[3]. Organismo nacional de acreditación. Servicios de acreditación de laboratorios de ensayo (2017) Consultado 03 de junio de 2020 en: https://onac.org.co/laboratorios-de-ensayo.

[4]. MINSALUD, Decreto 4725. Por el cual se reglamenta el régimen de registros sanitarios, permiso de comercialización y vigilancia sanitaria de los dispositivos médicos para uso humano, Bogotá DC, 2005.

[5]. ICONTEC, NTC 17025. Requisitos generales para la competencia de los laboratorios de ensayo y calibración, Bogotá DC: ICONTEC 2017.

[6]. ICONTEC, NTC 13485. Sistema de gestión de calidad. Dispositivos médicos, Bogotá DC: ICONTEC 2005.

[7]. MINSALUD, Resolución 4002. Por la cual se adopta el manual de requisitos de capacidad y almacenamiento de dispositivos médicos para uso humano, Bogotá DC, 2007.

[8]. Wang, B., Furst, E., Cohen, T., Keil, O. R., Ridgway, M. y Stiefel, R. Medical equipment management strategies. Biomedical Instrumentation \& Technology, 40(3), 233-237, 2006.

[9]. Enríquez, N., Álvarez, Y., Martínez, D. M., Pérez, A. y Lemgruber, A. Situación de la regulación de los dispositivos médicos en la Región de las Américas. Revista Panamericana de Salud Pública, 39(5), 238-244, 2016.

[10]. Zuckerman, D. M., Brown, P. y Nissen, S. E. Medical device recalls and the FDA approval process. Archives of internal medicine, 171(11), 1006-1011, 2011

[11]. ICONTEC, NTC IEC60601. Requisitos generales para la seguridad, Bogotá DC: ICONTEC 2001.

[12]. ICONTEC, NTC IEC 62304. Software para dispositivos médicos, Bogotá DC: ICONTEC 2006.

[13]. Llamosa, L. E., Contreras, L. G. M. y Lara, H. P. Fundamentos para el diseño de la prueba de seguridad eléctrica para equipo biomédico con base en la norma NTC-ISO-IEC-60601-1. Scientia et technica, 12(30), 321-326, 2006.

[14]. ICONTEC, NTC IEC 60601-1-8. Requisitos generales para la seguridad. Norma colateral: Requisitos generales, ensayos y guía para los sistemas de alarma en equipos electromédicos y sistemas electromédicos, Bogotá DC: ICONTEC 2005. 